\title{
A Heuristic Approach to Innovative Problem Solving
}

\author{
Bertil Hök* \\ *School of Innovation, Design and Engineering, Mälardalen University, Västerås, Sweden, and \\ Hök Instrument AB, Västerås, Sweden
}

\begin{abstract}
A methodic approach to innovative problem solving is suggested. First, Bayesian techniques are analyzed for quantifying, monitoring and predicting the process. The symmetry of Bayes' theorem implicates that the chances of success offrail ideas with small base rates can be boosted by highly accurate tests built on solid scientific ground. Second, a hypothesis is presented in which five methodic elements - connection, selection, transformation, balance and finish - are deemed to be necessary and sufficient to explain innovative solutions to complex problems. The hypothesis is supported by the analysis of disruptive innovations in several fields, and by emulation of a data base including 40,000 inventions. The reported findings may become useful in the further methodic development of innovative problem solving, especially in the risky and lengthy preconceptual phases.

Keywords: Innovative problem solving, heuristic reasoning, Bayes' theorem, methodic elements, innovation strategies.
\end{abstract}

\section{INTRODUCTION}

Disruptive innovations are invariably based on solutions to many-faceted problems, the roots of which are commonly traced to several scientific disciplines [1-6]. The importance of unconventional and cross-disciplinary thinking for innovative problem solving has been underlined by entrepreneurs, analysts and psychologists [7-9].

In this paper, a methodic approach to innovative problem solving issuggested. It is leaning heavily on the pioneering work by Pólya [10], highlighting the paradoxical nature of the mental processes involved. "The inventor's paradox" (ibid. p. 121-122) is classical: "The more ambitious plan may have more chances of success." The benefit of "thinking backwards" (ibid., p. 225-232) in addition to more conventional forward-striving was pointed out. Pólya also reintroduced heuristic reasoning (ibid. p. 113-114) fromthe old Greek; the use of rules of thumb or educated guesses to approach a problem, however,without de-emphasizing the importance of rigorous proof or scrutinizing validation of the final solution.

The methodic approach in this paper includes one probabilistic part enabling the stepwise progress towards a solution to be quantified and monitored. The second part is a hypothesis that five methodic elements are necessary and sufficient for solving even extremely complex problems. The methodic elements and theirunderlying analysis areshortly described with reference to literature and elementary facts. The results section includes observations concerning both the probabilistic and the hypothetical parts.Finally, the validity and the usefulness of the findings are discussed.

\section{METHODS}

The process of innovative problem solving may be viewed as a chain of events, as schematically depicted in Fig. 1.The starting pointcould coincide with the sudden revelation of an emerging barrier, or the problem may build up gradually through analytic or creative insight. This point is denoted as 'problem recognition'. It is commonly followed by further analysis and creative thinking in order to arrive at some idea on how to proceed. A step of major importance is to develop a working principlethat theoretically could carry the idea all the way to a solution.

A working principle should be realistic but may still be too immature to be tested against reality. A concept, on the other hand, should be a prototype of the final solution, although it may still have imperfections remaining to be resolved. The concept will be subjected to all sorts of tests, some of which will pass, whereas others may fail. Multiple redefining iterations on all previous levels may have to be carried out before an acceptable solution is reached. 


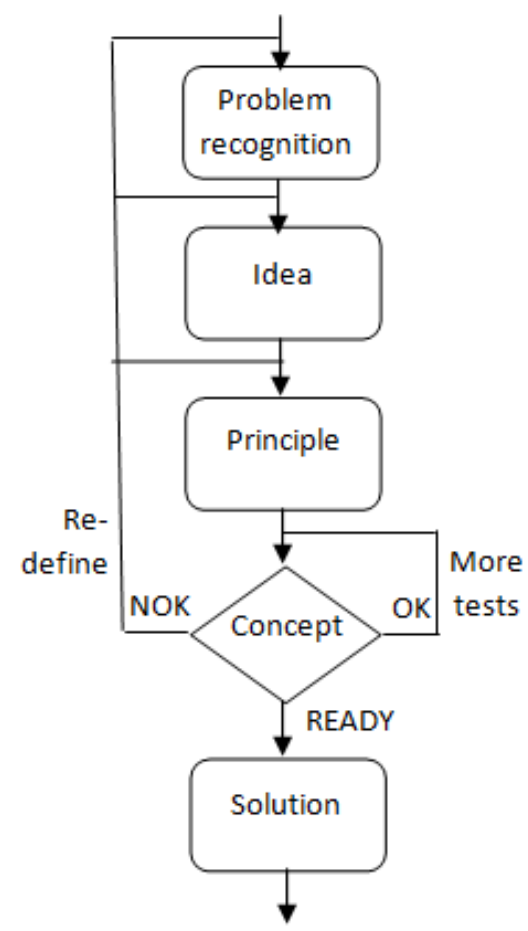

Figure 1. Flowgraph of innovative problem solving.

Innovative problem solving is a high-risk operation, and probabilistic methods should therefore be potentially useful. A methodic approach is believed to minimize risks and to improve the process efficiency.The classical Bayes' theorem[11] offers a unique possibility to quantify and monitor progress. It expresses the conditional probability $\mathrm{P}(\mathrm{A} \mid \mathrm{B})$ of an event $\mathrm{A}$ to be true when another event $B$ is true. $P(A \mid B)$ is uniquely defined by the individual probabilities $\mathrm{P}(\mathrm{A}), \mathrm{P}(\mathrm{B})$ and the reverse conditional probability $\mathrm{P}(\mathrm{B} \mid \mathrm{A})$ :

$P(A \mid B)=\frac{P(B \mid A) \cdot P(A)}{P(B)}$

If $\mathrm{P}(\mathrm{A})$ represents the base rate, or initial probability of reaching the solution to a problem, and $\mathrm{P}(\mathrm{B})$ is the probability of a test result to be true, then $\mathrm{P}(\mathrm{A} \mid \mathrm{B})$ will express the expected progress due to the test.

In addition to the work breakdown structure outlined in Fig. 1, thetask of problem solving is divided into five methodic elements, the usefulness of which will depend on the character of the problem, and by the position within the process according to Fig. 1. A short description of each element is given below.

Connection of hitherto unconnected aspects related to the problemis the first element. It has the power of resolving inconsistencies by introducing new perspectives to the problem. According to psychologists,human creativity is related to theability of the brain to establish connections betweenremote memory areas $[8,12]$. To problem solvers, the 'aha' or 'eureka' experience may be synonymous with an inventive step, and this notion is even recognized by legislators. For an invention to be patentable, it should not 'be obvious to a person skilled in the art' [13]. The discovery of unexpected connections seems to be at least partly subconscious [9, pp. 24-25] but is commonly combined with analytical reasoning [9, pp. 66-67].

Selectionis in many ways complimentary to connection. It brings an issue to its head, and itsets a limit to the seemingly endless connections and associations. It is useful for finding a critical item among a large amount of data, to divide a complex problem into manageable subtasks, and to focus on the most important ones. Whereas connection is related to the mathematical operations of addition and integration, selection is linked to subtraction and differentiation.

Transformation is a mapping operation from one domain into another.In applied mathematics, it may be exemplified by the Fourier and Laplace transforms. The human brain is able to transform physical objects into mental images via sensory receptors, and further processed at higher cognitive levels. Size, position, shape, character, color, time/frequency, are a few examples of transformable domains. Transformation may be an iterative, multistage process, and it is scalable from local to global dimensions, from neural topsychological behavior, from technological to social, and even political domains.

Balance has to be employed when dealing with inseparable opposites (black/white, plus/minus, male/female, etc.). When circumstances are changing with time, active balancing using feedback control needs to be employed. Ultimately, the multitude of aspects in a complex problem situation requires a balanced view.

Finish is a unique element in which a concept is converted into a complete solution verified and validated by testing and refining until all the prescribed requirements of the solution are fulfilled.

The hypothesis put forward in the introductionthat the five elements are necessary and sufficient for innovative problem solvinghas been subjected to two separate investigations, both being examples of 'reverse engineering'. The first investigation seeks to examine timing properties and to find heuristic explanationsin view of the methodic elements to some disruptive innovations in science, technology and politics, more specifically the general theory of relativity $[14,15]$, the disclosure of the DNA molecular structure [16], the abolition of apartheid [17], the invention of the world wide web [18], and the iPhone innovation [19]. 
The second investigation is based on data obtainedfromAltshuller et al [20], commonly referred to as the TRIZ method (Russian acronym, freely translated as the theory of inventive problem solving).The 40 solution principles of Altshuller's contradiction matrix assembled from a data base of 40000 inventions (ibid. p. 261-276)may be assigned and reduced to the five methodic elements,allowing the incidence and links between them to be quantified.

\section{RESULTS}

An alternative expression of Bayes' theorem (eq. (1)) following directly from basic probability definitionsis this equation:

$$
P(A \mid B)=\frac{P(B \mid A) \cdot P(A)}{P(B \mid A) \cdot P(A)+P(B \mid C A)) \cdot P(C A))}
$$

By $\mathrm{CA}$ is meant the complimentary of $\mathrm{A}$. Equation (2) was used to generate the graph shown in Fig. 2, in which $\mathrm{P}(\mathrm{A} \mid \mathrm{B})$ is plotted against the base rate $\mathrm{P}(\mathrm{A})$ with three parameter settings of $\Delta=\mathrm{P}(\mathrm{B} \mid \mathrm{A})$ $\mathrm{P}(\mathrm{A})$.

The $S$ shape of the curves is more pronounced at small values of $\Delta$, and illustrates the non-linearity appearing at small and big base rates. When $\mathrm{P}(\mathrm{A}) \rightarrow 0$ the slope of the curves increases with increasing $\Delta$.

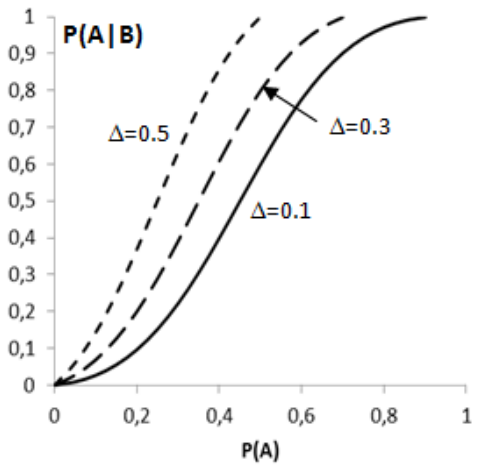

Figure 2. The three S-shaped curves directly follow fromBayes' theorem assuming a constant difference $\Delta=\mathrm{P}(\mathrm{B} \mid \mathrm{A})-\mathrm{P}(\mathrm{A})$.

It is observed that the curves are symmetric to rotation $180^{\circ}$. This is a direct consequence of the symmetric nature of Bayes' theorem with respect to substitution between the arguments $\mathrm{A}, \mathrm{B}$, and their complimentaries.A further consequence is this: to achieve a conditional probability $\mathrm{P}(\mathrm{A} \mid \mathrm{B})>50 \%$, the base rate $\mathrm{P}(\mathrm{A})$ must be matched with a test probability $\mathrm{P}(\mathrm{B} \mid \mathrm{A})>(1-\mathrm{P}(\mathrm{A}))$.

The slightly modified version of eq. (2)

$$
P(A \mid B)=\frac{1}{1+\frac{P(B \mid C A) \cdot P(C A)}{P(B \mid A)-P(A)}}
$$

illustrates a related observation:

When

$\mathrm{P}(\mathrm{B} \mid \mathrm{C} \mathrm{A})<<\mathrm{P}(\mathrm{A})$, then $\mathrm{P}(\mathrm{A} \mid \mathrm{B})$ will approach unity asymptotically. A numerical example: If the base rate $\mathrm{P}(\mathrm{A})=1 \%, \mathrm{P}(\mathrm{CA})=99 \%, \mathrm{P}(\mathrm{B} \mid \mathrm{A})=99.99 \%$ and $\mathrm{P}\left(\mathrm{B} \mid \mathrm{C}^{\mathrm{A}}\right)=0.01 \%$, then $\mathrm{P}(\mathrm{A} \mid \mathrm{B})=99.02 \%$.

The risk exposure of the early phases of innovative problem solving is highlighted by the approximate durations of the preconceptual and finishing phases of the investigated disruptive innovations [14-19]depicted in Fig. 3. The total duration ranged from 8 to 33 years, and the average of the preconceptual phases were $89 \%$ of the total duration.

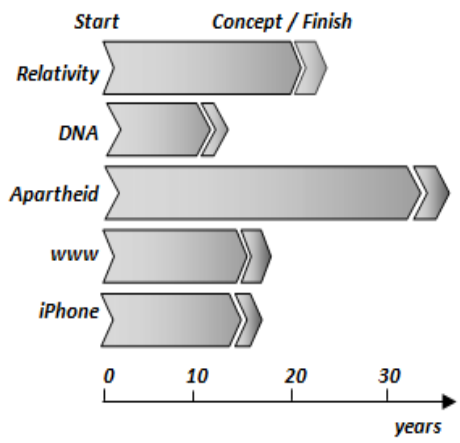

Figure 3. The approximate duration of the preconceptual and finishing phases of the investigated innovations.

The methodic elements described in the method section were combined to provide heuristic explanations of the disruptive innovations. Some examples are given below.

In Einstein's general theory of relativity, the entities of time and space were connected into a nonEuclidean, four-dimensional space-time, resolving inconsistencies related to Newtonian gravity and inertia. The consistency of the theory was secured by the mathematical transformationsdescribing the detailed interdependence ofspace, time, matter, and energy.

The disclosure of the DNA molecular structure was enabled by a transformation process from the observed X-ray diffraction pattern generated by a DNA specimen into the double helix molecular structure in which the genetic code is stored in a replicable fashion.

In his struggle for human rights and against apartheid, Nelson Mandela successfully adopted a strategy of active balancing, ranging from militant action to reach awareness, via imprisoned passive resistance to gain acceptance, and finally, reconciliation to reach the stage of demand.

For the world wide web invented by Tim Berners-Lee, a surprisingly small number of items - 
the universal resource identifier, the hypertext transfer protocol, and the hypertext markup language - were selected, interconnected and transformed into a unified concept which revolutionized the communication and management of digitized information.

The iPhone innovation included several interconnected items, the multi-touch screen and related software in particular, for the disruptive transformation of the cell phone into an attractive multipurpose tool and toy, fitting into everyone's pocket.

Table 1 is a summary of the extent to which the methodic elements explainsome exceptional problem-solving achievements.

Table 1. The major (X)or minor (x) extent to which exceptional achievements can be explained by the methodic elements connection (C), selection (S), transformation (T), balance (B).

\begin{tabular}{|c|c|c|c|c|c|}
\hline Exceptional achievement & $C$ & $S$ & $T$ & $B$ & $F$ \\
\hline General theory of relativity & $\mathbf{X}$ & $\mathrm{X}$ & $\mathbf{X}$ & $\mathrm{x}$ & $\mathrm{X}$ \\
\hline DNA molecular structure & $\mathrm{X}$ & $\mathrm{X}$ & $\mathbf{X}$ & $\mathrm{X}$ & $\mathrm{x}$ \\
\hline Abolition of apartheid & $\mathbf{X}$ & $\mathrm{X}$ & $\mathrm{x}$ & $\mathbf{X}$ & $\mathbf{X}$ \\
\hline World Wide Web innova & $\mathbf{X}$ & $\mathbf{X}$ & $\mathbf{X}$ & $\mathbf{x}$ & $\mathbf{x}$ \\
\hline The iPhone innovation & $\mathbf{X}$ & $\mathrm{x}$ & $\mathbf{X}$ & $\mathrm{x}$ & $\mathbf{X}$ \\
\hline
\end{tabular}

The small ' $x$ 's in Table 1 indicate that these methodic elements were actually influential, but to a minor degree.For example, in the Einstein case, there was a selection towards macrocosmic phenomena rather microcosmic, a balance between collegial involvement or not, and adramatic finish. But the influence of these elements on the total outcome was not decisive. Correspondingly, it is possible to trace some aspects of all methodic elements also in the other cases of the investigation.

Fig. 4schematically illustrates how certain elements are associated with different phases of the problem-solving process.

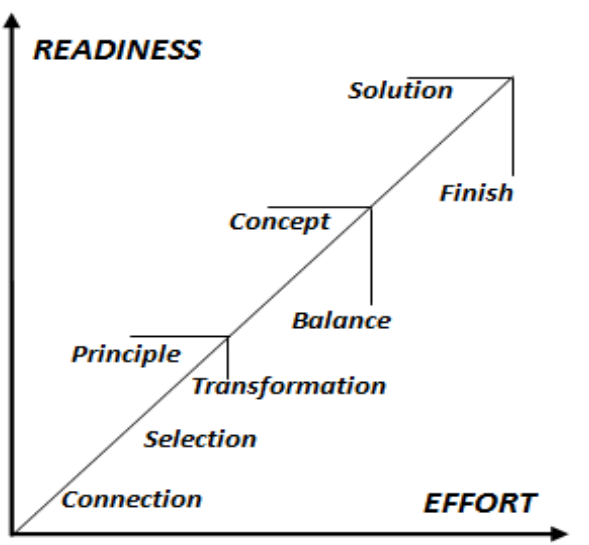

Figure 4. Schematic relation between solution readiness and effort positioning the major usefulness of the methodic elements.
Connection and selection are primarily used in the pre-principle phase to resolve inconsistencies. Transformation is a crucial element for refining a principle into a viable concept. The balancing element is useful for bridging quantifiable gaps, and the finishing element is required to close all remaining issues until a complete solution exists.

The TRIZ method [20] is based on the analysis of 40,000 inventions using a 39 x 39 matrix of improved characteristics to identify the action of 40 solution principles. In the present study, each one of Altshuller's 40 principles has been attributed to one of the five methodic elements according to Table 2 .

Table 2. Assignment of the TRIZ solution principles [20] to the methodic elements connection (C), selection (S), transformation (T), balance (B) and finish $(\mathrm{F})$.

\begin{tabular}{|l|l|c|c|c|c|c|}
\hline & \multicolumn{1}{|c|}{ TRIZ principles } & C & S & T & B & F \\
\hline 1 & Segmentation & & X & & & \\
\hline 2 & Extraction & & X & & & \\
\hline 3 & Local Quality & & X & & & \\
\hline 4 & Asymmetry & & X & & & \\
\hline 5 & Consolidation & & & & & \\
\hline 6 & Universality & X & & & & \\
\hline 7 & Nesting (Marioshka) & & & & X & \\
\hline 8 & Counterweight & & & X & & \\
\hline 9 & Prior counteraction & X & & & & \\
\hline 10 & Prior action & X & & & & \\
\hline 11 & Cushion in advance & & & & X & \\
\hline 12 & Equipotentiality & & & X & & \\
\hline 13 & Do it in reverse & & & X & & \\
\hline 14 & Spheroidality & & & X & & \\
\hline 15 & Dynamicity & & & X & & \\
\hline 16 & Partial/excessive action & & & & X & \\
\hline 17 & Translating, dimension & & & X & & \\
\hline 18 & Mechanical vibration & & & X & & \\
\hline 19 & Periodic action & & & X & & \\
\hline 20 & Continuity of action & & & X & & \\
\hline 21 & Rushing through & & & & & \\
\hline 22 & Convert harm/benefit & & & X & & \\
\hline 23 & Feedback & & & & X & \\
\hline 24 & Mediator & & & & & \\
\hline 25 & Self service & & & & & \\
\hline 26 & Copying & & & X & & \\
\hline 27 & Dispose & & & X & & \\
\hline 28 & Replacement & & & X & & \\
\hline 29 & Pneumatic/hydraulic & & & & \\
\hline 30 & Flex films, membranes & & & X & & \\
\hline 31 & Porous materials & & & & \\
\hline 32 & Changing color & & & \\
\hline 33 & Homogeneity & & & & \\
\hline 34 & Reject, regenerate & Transformation & & & \\
\hline 35 & Phase transition & Thermal expansion & & & \\
\hline 37 & Accelerated oxidation & & & \\
\hline 39 & Composite materials & & & \\
\hline
\end{tabular}


The result from the TRIZ analysis is summarized in Fig. 4, showing the relative incidence of each of the methodic elements, and their crosslinks based on data obtained from Altshuller et al [20]. Both incidence and cross-links are dominated by the transformation and finishing elements, whereas the role of the balancing element is considerably weaker.

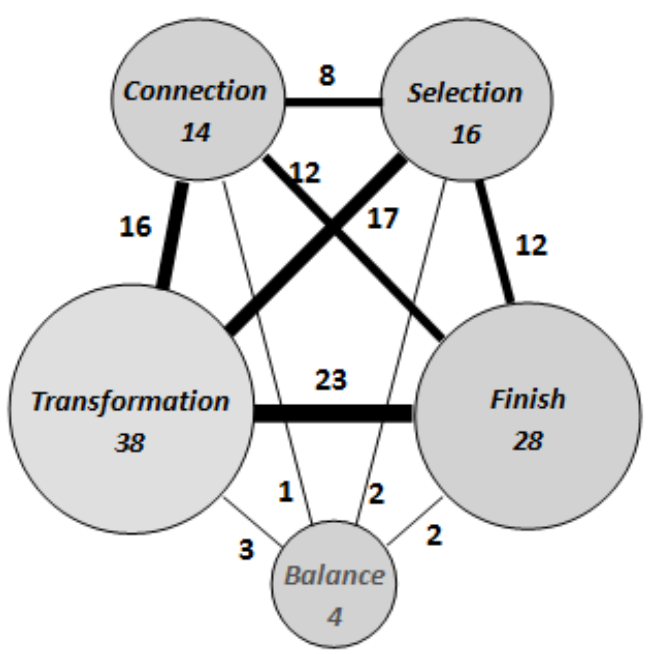

Figure 5. Relations between the five methodic elements with respect to incidence (numbers within the circles expressed as \% of total) and cross-links (numbers at the respective intersecting lines, also expressed as \% of total). Original data obtained from Altshuller et al [15].

\section{DISCUSSION}

The observations concerning Bayes' theorem provide a strong indication for the usefulness of Bayesian techniques in quantifying, monitoring and predicting the progress of innovative problem solving processes. The symmetric nature of Bayes' theorem, equation (3), and the numerical example demonstrate that a test resting on solid scientific ground, and therefore having an extremely small rate of false positives, can indeed override the influence of the base rate. This fact is of importance in all phases of innovative problem solving, although it maysound contradictory tothe 'base rate fallacy' taught by psychologists; the human tendency to trust anecdotes more than statistics [8, p. 146-155].

The hypothesis put forward in the introduction is supported by the analysis of some disruptive innovations in various fields, indicating that heuristic explanations based on the five methodic elements can be given, evento the solutions of such complex problems.

As indicated in Fig. 4, the connective element is primarily useful in the early phases of the problem-solving process, and in the presence of barriers which are difficult to quantify. A selection process may divide a highly complex problem into separate and manageable parts, enabling the most critical ones to be isolated from irrelevant disturbances. A creative transformation may, like an unexpected connection, resolve barriers of inconsistency. The balancing element represents an "emergency exit" to manage inseparable and opposing units. During the finishing process, all aspects of the project need to be tied together into a unity. In the final stage, the perspective should be turned inside out, starting to develop the consequences of an existing solution.

From the emulation of TRIZ data [20] summarized in Fig. 5, it is concluded that the transformation and finishing elements are dominating within the data base of $\operatorname{mid} 20^{\text {th }}$ century inventions in the fields of mechanical and electrical engineering. This is consistent with expectations in view of the fact that most of the inventions in the data base were incremental, rather than disruptive.

The hypothesis brought forward in this paper will remain a hypothesis until more substantial evidence has been gathered. The findings may potentially become useful to innovative problem solvers, especially in the early stages of development. The importance of improved methodic development directed towards the preconceptual phases is underlined by Fig. 3, illustrating the dominance of these phases in the overall duration.

\section{CONCLUSION}

It is concluded that Bayesian techniques are useful for quantifying, monitoring and predicting innovative problem solving processes. It follows from Bayes' theorem that a test with extremely small rate of false positives may override a small base rate. The hypothesis that five methodic elements connection, selection, transformation, balance and finish - are necessary and sufficient may become useful in the further methodic developmentof innovative problem solving, especially its risky and lengthy preconceptual phases.

\section{ACKNOWLEDGEMENTS}

The author gratefully acknowledges the inspiration from numerous colleagues and friends, in particularthe valuable comments on the manuscript byLasse Tenerz, Håkan Pettersson, and Henrik Rödjegård.

\section{REFERENCES}

[1] R. Christensen, The innovator's dilemma(Boston, MA, Harvard Business School Press, 1997).

[2] T. Kelley, The art of innovation(New York, NY, Random House, 2001).

[3] V Govindarajan, C. Trimble, Ten rules for strategic innovators(Boston, MA, Harvard Business School Press, 2005). 
[4] W. C. Kim, R. Auborgne, Blue ocean strategy(Boston, MA, Harvard Business School Press, 2005).

[5] G. Faltin Kopf schlägt Kapital(München, Germany, Hanser, 2008).

[6] B. Hök, L. Tenerz, R. Stubbe, Inside innovation (Stockholm, Sweden, Bookhouse, 2011).

[7] T. Kelley, J. Littman, The ten faces of innovation(New York, NY, Random House, 2006).

[8] D. Kahneman, Thinking, fast and slow (New York, NY, Farrar, Straus and Giroux, 2011).

[9] J. Kounios, M. Beeman, The eureka factor(New York, NY, Random House, 2015).

[10] G. Pólya, How to solve it ( $2^{\text {nd }}$ Ed., New York, NY, Doubleday Anchor Books, 1957).

[11] T. Bayes, An essay towards solving a problem in the doctrine of chances, Phil. Trans. 53 (1763), 370-418.

[12] S. Mednick, The associative basis of the creative process, Psychological Review, vol 69, 1962, 220-232.

[13] The European Patent Convention, Part II, Chapter 1, Article 56, Inventive step.

[14] A. Einstein, How I created the theory of relativity, Kyoto Lecture, December 14 1922, translated by Y. A. Ono, Physics Today, August 1982.

[15] A. Einstein, The meaning of relativity, Princeton, NJ, Princeton University Press, $1922,6^{\text {th }}$ Ed. 1956.

[16] J. D. Watson, The double helix (London, UK, Weidenfeld\& Nicolson, 1968).

[17] N. Mandela, Long walk to freedom (London, UK, Little, Brown \& Co, 1994).

[18] T. Berners-Lee, Weaving the web (New York, NY, Harper Collins, 1999).

[19] W. Isaacson, Steve Jobs - the authorized biography (New York, NY, Simon \& Schuster, 2011).

[20] G. Altshuller, The innovation algorithm, translated and edited from Russian by L. Shulyak and S. Rodman, Worcester, MA, Technical Innovation Center, 2007. Original Russian text published in 1973. 